\title{
Discussion: Influence of layer bonding on the prediction of pavement life
}

\section{K. Khweir and D. Fordyce}

\section{B. Hakim, Scott Wilson Pavement Engineering}

I have three comments on the above paper.

My first comment relates to pavement evaluation methods. The paper suggests that the capabilities of the falling weight deflectometer (FWD) are limited, and the authors alternatively propose using laboratory-measured bituminous stiffnesses from core samples and the description of good foundation to predict the pavement life. It is not clear from the paper how the California bearing ratio (CBR) values of 30 and 15\% quoted for the sub-base and subgrade were measured, or how stiffness values of 300 and $150 \mathrm{MPa}$ were predicted for the sub-base and subgrade respectively.

The FWD has been a major contributory factor for the development of pavement engineering as a discipline in the last two decades. This is due largely to its ability to test the in-situ pavement under realistic loading, and to measure the response to a high degree of accuracy. However, additional information from surveys such as coring, ground-probing radar (GPR) and the dynamic cone penetrometer (DCP), combined with materials testing, is usually required to assist in pavement analysis and materials characterisation. It should be noted that the laboratory stiffness measures individual intact pavement layers of a core sample from a specific location, whereas the FWD describes the in-situ effective stiffness of the various pavement layers incorporating cracks, voids and other defects. ${ }^{27}$ Additionally, the foundation stiffness under the confined pavement condition can be computed from FWD data to describe the material nonlinearity under appropriate stress levels. Hence the use of the FWD and associated techniques can provide more accurate results than the method proposed by the authors for analytical/mechanistic pavement evaluation.

Secondly, in relation to bond assessment methods, in section 1 of the paper the authors quote ranges of bond stiffness from my works to describe the bonding condition between pavement layers. These values represent the bond stiffness at the interface in a multi-layered elastic pavement system under traffic loading. The bond stiffness can be back-analysed from FWD data for use in both pavement evaluation and design. ${ }^{28,29}$ Other test methods use alternative procedures and apply different stress conditions, which can result in other bond values. The pull-off test measures the tensile bond strength, the torque test measures bond strength under a torque force, and the direct shear test measures bond strength and stiffness under a shear force in the laboratory.

In their conclusion the authors recommend use of the torque test on cores extracted from the pavement to determine the bond strength at the interface, without defining acceptable values relating to performance and how the test results can be used in pavement design and assessment of residual life. Practical evidence indicates that, if poor or medium bond exists between bituminous layers, they will be separated under the torque force applied by the rotary core barrel during coring, and therefore the torque test will not be possible. Hence the use of FWD data to back-analyse the bond stiffness between layers using a specialised program, such as BONDBACK, ${ }^{28,29}$ is recommended. The results represent fundamental engineering values to describe the bond stiffness under traffic loading for use in the analytical/mechanistic method of pavement evaluation and design. This method of bond assessment using the FWD, supplemented by other testing techniques, has been successfully used in the UK and overseas for both airport and highway pavements, and has produced results consistent with observed pavement behaviour.

My third comment relates to pavement life prediction. The authors used a laboratory fatigue line for the bituminous material multiplied by a shift factor of 77 to account for in-situ condition, with some concern. The in-situ fatigue properties will depend on the history of the material, which has been subjected to different stress levels, temperature variations, loading magnitude and frequency, and also has to account for the possible healing nature of the bituminous material. Therefore I propose that a suitable approach is to compare the laboratory-predicted fatigue line for site materials with the fatigue line of a 'standard' dense bitumen macadam material prepared in the laboratory. The measured shift factor, if any, can then be applied to an in-situ standard calibrated line (such as that presented in TRL report LR1132 ${ }^{19}$ ) and used in the pavement life prediction.

Additionally, the effect of poor bond on pavement deterioration and failure modes should be considered. Fatigue cracking was traditionally assumed to initiate under traffic 
loading at the bottom of the bituminous layer and propagate towards the surface. However, more recent work suggests a different deterioration mechanism for thicker pavements, namely surface-initiated cracking.

Assuming that the classical pavement failure modes apply for thinner pavements, tensile strains at the bottom of each bituminous layer need to be calculated if the effect of poor bonding is to be taken into account (as the layers will act independently). Therefore, for two poorly bonded layers, two cracks may initiate and propagate within the bituminous material under traffic loading, causing faster pavement deterioration (see Fig. 7). On the other hand, if the crack starts from the surface and propagates towards a debonded interface, it might extend horizontally causing further slippage, but not extend into the lower layers (see Fig. 8).

However, the above scenarios assume that the bond stiffnesses between bituminous layers stay the same during the pavement life and are not sensitive to temperatures. Previous work ${ }^{28}$ has suggested that, based on limited in situ testing:

(a) a combination of traffic and higher temperature will improve the bond stiffness

(b) age alone did not seem to improve the poorly bonded layers

(c) sections with very poor bond did not appear to improve with time or traffic.

\section{Authors' reply}

In response to the discusser's first point, the FWD has contributed to pavement engineering significantly. The paper highlighted the flaws with the some of the methods of analysis that use deflections values from FWD surveys. The description of the capabilities of an FWD are limited because the FWD gives a measure of pavement stiffness but the analysis becomes more approximate as it is used to extract more details. Analysis based on FWD surveys cannot be completed without extra information, such as:

(a) layer thickness, such as from a GPR survey

(b) foundation assessment, such as DCP testing

(c) coring process-verification of the effective thickness, stiffness modulus, interface bonding and generally the integrity of the bound layer.

However, if the asphaltic layers are taken as a multi-layer system with no provision for the friction between the layers in back-calculation of the FWD, and knowing that the interlayer bond is unable to provide an acceptable limit of bond strength, the analysis would be flawed. The paper is criticising the culture of reducing the stiffness modulus of the bound layers to account for low interface bond. It is often believed that, during FWD analyses, pavement layers with no bond are compensated for by a lower stiffness modulus than the absolute value of each individual material forming the layer system, in order to meet the recorded site deflection value. This belief has a shortcoming in that decreasing the stiffness modulus will increase the strain value at the bottom of the lower layer, whereas using a high stiffness modulus with no or reduced interface friction will increase the strain value at the bottom of the upper layer (above the interface). Also, it is very important for any analysis based on an FWD survey to take into account the effective layer thickness, effective stiffness modulus and

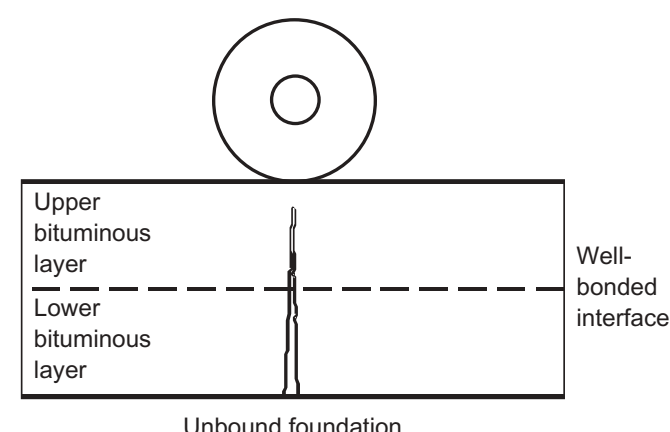

Unbound foundation

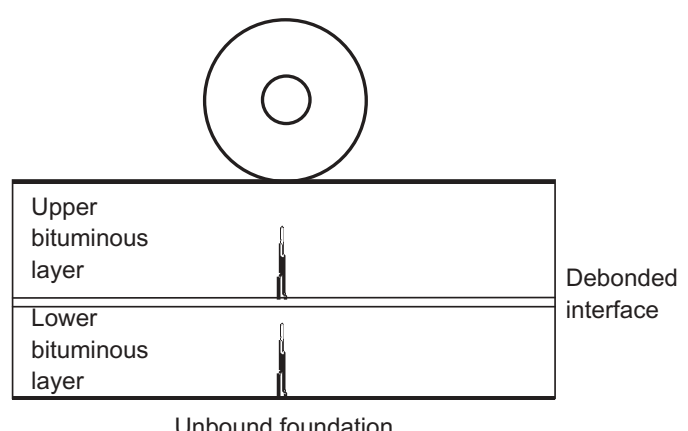

Unbound foundation

Fig. 7. Effect of debonding of bituminous layers on crack initiation and propagation for classical pavement deterioration
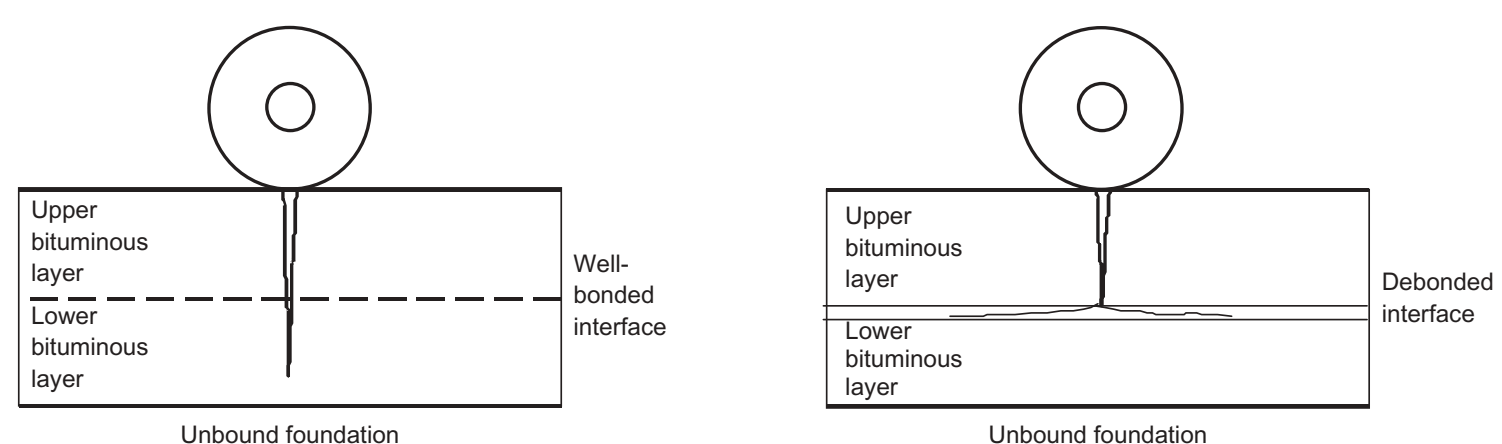

Unbound foundation

Unbound foundation 
interface bonding condition. The paper is not criticising the FWD apparatus or the principle on which it is based, but some practices used with analysis of the deflection data and in consequence any conclusion based on these practices are questioned.

In relation to the discusser's second point, it is stated in the paper that assessment of the interface bond can be carried out by many bond tests as part of compliance testing. Ideally this can be assessed through the construction of a trial panel prior to the construction process to verify the process. Nevertheless, any interface that survives the coring process would not be far from the acceptable limit. The British Board of Agrément has published a convenient procedure for testing the interface of the surfacing layer as part of HAPAS approval. This method can be modified to test all pavement layers. At present there is no testing protocol that would yield data that can be used directly in the analysis.

The conclusion of the paper did not recommend the torque test.

Taking the discusser's final point, the fatigue shift factor was used to bring the laboratory data to the site performance level, where the pavement life reaches critical condition. It was stated clearly that the authors have no particular faith in the shift factor of 77 for the reported case study; this factor happens to create superposition of the fatigue line reported by TRRL LR 1132. ${ }^{19}$ Instead of using a shift factor another approach can be adopted to convert the laboratory life to site life. This approach compares the laboratory fatigue life of cores taken from the investigated site with a typical laboratory fatigue line generated with a similar mixture. Such a comparison can lead to a displacement and rotation of the fatigue profile of the investigated site. The typical fatigue profile is comparable with the typical profile defined from site experience created by TRL LR 1132; the difference is essentially a shift in the profiles. Adjusting the TRL profile using the same displacement and rotation as defined within the laboratory-created data can define an actual site fatigue profile for the investigatory site.

The typical fatigue profile and the fatigue profiles generated from the investigated site are both laboratory data that are based on the same mode of testing using similar testing

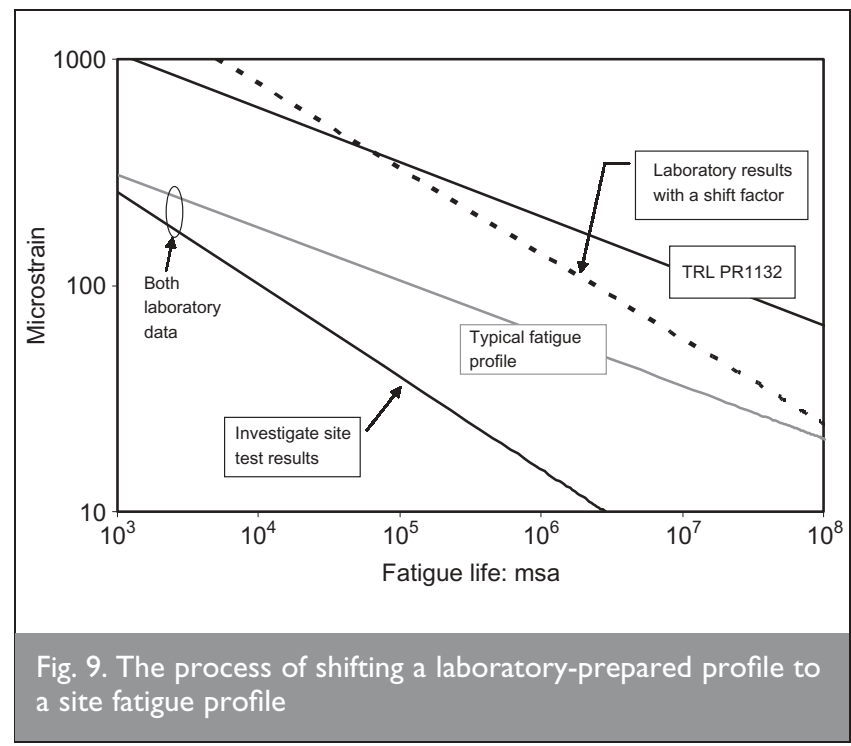

apparatus. As both sets of data are comparable, ratios of fatigue lives were determined at each strain level. TRRL LR 1132 is regarded as a typical site fatigue profile for all DBM material. This fatigue profile was adjusted by the above ratio. This adjustment can be carried out by dividing the fatigue life from the fatigue profile of TRRL 1132 by the above ratios at the same strain levels used to calculate these ratios. The product of this calculation can be plotted to generate a new fatigue profile (see Fig. 9). This new profile can then be used to calculate the life of the investigated pavement. This protocol can be considered as the closest approach to evaluating the fatigue properties of an investigated pavement. But to assume that the shift of a badly constructed pavement is to shift similarly to a typical pavement may raise a concern.

Other information raised in the third comment with regard to the crack development of a pavement layer system is very useful data, and the authors are in agreement with this understanding. However, not all thick pavements would crack top down; only well-constructed thick pavements may do so. On many occasions thick pavements have failed prematurely in spite of the surface layer showing no distress or cracking, particularly on a newly constructed pavement. Many cored pavements show the lower base layer to have loose coated aggregate, or to have disintegrated upon coring (see Fig. 10).

\section{N. H. Thom, University of Nottingham}

As the University of Nottingham is currently researching the issue of layer bonding on behalf of the Highways Agency, I feel some comments are in order.

(a) I should like to give general support to the authors in drawing attention to what is an important issue, and one that is poorly understood.

(b) It is certainly true that reliance on a conventionally backcalculated FWD stiffness value is not appropriate in a case where layers are poorly bonded. However, I should like to draw attention to work by Hakim, ${ }^{8}$ who successfully backcalculates a bond stiffness. This seems to be a most useful non-destructive technique, allowing realistic layer stiffnesses to be obtained as well as a usable measure of bond quality.

(c) I believe caution is required in using the same relationship between calculated tensile strain in an asphalt and life to failure in a debonded case as for a fully bonded case. These

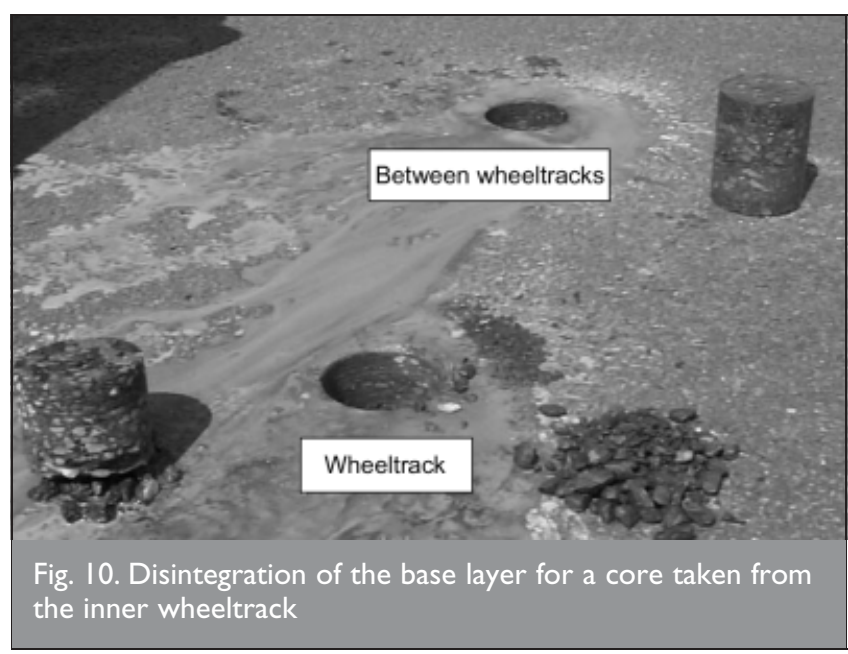


relationships are really empirically based and take no account of the different crack propagation process that would be expected in a crack initiating at an interface.

(d) The authors mention various bond strength measurement techniques but omit the Leutner test. This is a highly practical test developed in Germany, in which a cored specimen is sheared at an interface at a controlled rate (the same rate as in a CBR or Marshall test). It is being investigated in some detail, together with the torque test, in the current University of Nottingham research project 'Influence of layer bonding on the prediction of pavement life'.

\section{Authors' reply}

(a) The authors appreciate the support of Dr Thom and his contribution to the discussion of this important subject. This paper was written around 2 years ago, and it reflects the thinking and the information available at that time.

(b) The use of the FWD with the right approach in the analysis, taking into account the effect of interface bonding, effective stiffness and effective thickness, certainly makes it the most useful non-destructive technique for pavement evaluation. Defection values would predict any of the above parameters if the rest or some of these parameters were known.

(c) It is quite right to differentiate between bonded and debonded pavements so far as the process of crack propagation is concerned. An empirical relationship to calculate life was used to highlight the problem of debonding. However, the process of life prediction is always difficult; nevertheless, any modification to the calculation procedure would reduce the life even further. In other words, the influence of debonding would be greater.

(d) We are looking forward to the work currently being carried out at Nottingham University with great interest in regard to the measurement of bond strength. The paper highlighted some of the methods used to evaluate the bond strength, but by no means was it intended to be a comprehensive review of all the test methods. The Leutner test is another test that can be used for bond strength evaluation, particularly if the current research at Nottingham University is concluded successfully with regard to the practicality of the test. Also, at Pavement Technology Ltd at Heriot-Watt University, a bond strength test has been developed for interface evaluation. The test measures the shear strength at the layer interface for cores taken horizontally at the interface. The test is already used for interface evaluation of many pavements with hydraulically stabilised binders.

\section{REFERENCES}

27. Hakim B., Brown S. F. and Armitage R. J. Pavement evaluation and strengthening design: sixteen years' experience. Proceedings of the 9th International Conference on Asphalt Pavements, Copenhagen, 2002.

28. Hakim B., Cheung L. W. and Armitage R. J. Use of FWD data for prediction of bonding between pavement layers. International Journal of Pavement Engineering, 1999, 1, No. 1, 49-59.

29. HAKIM B. The importance of good bond between bituminous layers. Proceedings of the 9th International Conference on Asphalt Pavements, Copenhagen, 2002. 